$12-26-2011$

\title{
A critical analysis of the current state of neurosurgery training in Pakistan
}

Muhammad Shahzad Shamim

Aga Khan University, shahzad.shamim@aku.edu

Muhammad Zubair Tahir

Aga Khan University, zubair.tahir@aku.edu

Saniya Siraj Godil

Aga Khan University

Rajesh Kumar

Aga Khan University

Arshad Ali Siddiqui

Aga Khan University

Follow this and additional works at: https://ecommons.aku.edu/pakistan_fhs_mc_surg_neurosurg

Part of the Neurology Commons, Neurosurgery Commons, and the Surgery Commons

\section{Recommended Citation}

Shamim, M., Tahir, M., Godil, S., Kumar, R., Siddiqui, A. (2011). A critical analysis of the current state of neurosurgery training in Pakistan. Surgical Neurology International, 2, 183.

Available at: https://ecommons.aku.edu/pakistan_fhs_mc_surg_neurosurg/12 


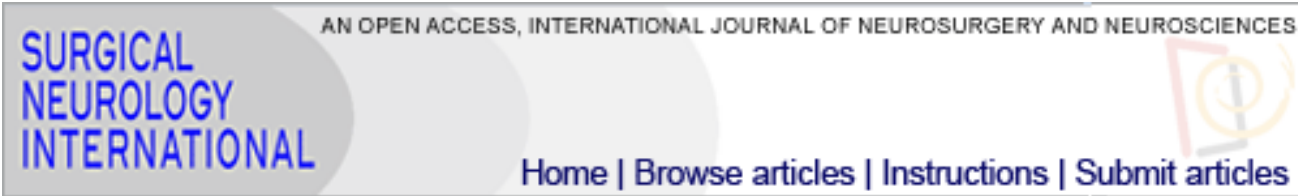

Surg Neurol Int. 2011; 2: 183

Published online 2011 Dec 26. doi: 10.4103/2152-7806.91138

\section{A critical analysis of the current state of neurosurgery training in Pakistan}

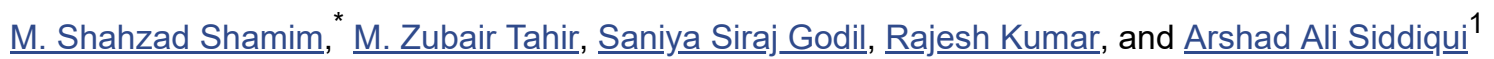

Section of Neurosurgery, Department of Surgery, Aga Khan University Hospital, Karachi, Pakistan

${ }^{1}$ Department of Neurosurgery, Jinnah Postgraduate Medical Center, Karachi, Pakistan

M. Shahzad Shamim: shahzad.shamim@aku.edu; M. Zubair Tahir: muhammad.zubair@aku.edu; Saniya Siraj Godil: saniyasiraj@hotmail.com; Rajesh Kumar: rajesh.kumar@aku.edu; Arshad Ali Siddiqui: doc4brains@yahoo.com

Corresponding author

Received 2011 Feb 18; Accepted 2011 Nov 21.

Coppyright : @ 2011 Shamim MS.

This is an open-access article distributed under the terms of the Creative Commons Attribution License, which permits unrestricted use, distribution, and reproduction in any medium, provided the original author and source are credited.

This article has been cited by other articles in PMC.

\section{Abstract}

\section{Objective:}

To observe interdepartmental variation in the availability of resources and academic activities within the various neurosurgery programs of Pakistan.

\section{Methods:}

This was a proforma-based survey of neurosurgery trainees and young neurosurgeons of Pakistan, looking at the academic infrastructure and output of their programs. The proforma was filled by 36 respondents from 11 neurosurgery centers of the country. All these centers were accredited for neurosurgery training in Pakistan.

\section{Results:}


Out of the 36 respondents, 30 were completing a Fellowship training (FCPS) and six were enrolled for a Master in Surgery (MS) program. About $80 \%$ of the participants used the Youman's Textbook of Neurosurgery as a reference book. Only $40 \%$ of the candidates had access to more than one indexed neurosurgery journal. Structured academic sessions (e.g., journal clubs and neuropathology meetings) were lacking in a majority of the training institutes, $95 \%$ of the trainees had no microsurgical laboratory experience, and modern neurosurgical tools (frameless neuronavigation system, neuroendoscopy) were in use at a few centers only.

\section{Conclusion:}

Neurosurgery training in Pakistan is not uniform and wide variations exist between the programs at the centers evaluated. We recommend exchange programs between centers at national and international levels, to allow trainees to gain first-hand exposure to training components not available in their own center.

Keywords: Neurosurgery, Pakistan, programs, training

Neurosurgery is one of the younger surgical specialties.[15] It is, however, one of the most dynamic specialties with major changes observed in the past few years.[11] In Pakistan, modern neurosurgery began to evolve about six decades ago, and neurosurgical training has developed tremendously since then.

Pakistan is a developing country with a literacy rate of $56 \%$, estimated by a survey conducted by the Ministry of Education in $2004-2005$, and a gross enrollment rate of $86 \%$ at the primary level, $46 \%$ at the middle level, and $44 \%$ at the secondary level. The total expenditure on education is only $2.21 \%$ of the gross domestic product (GDP). This low expenditure on education contributes greatly to the difficulties encountered in running higher educational programs in the country, as most of the budget is spent on primary education.

Today, there are an estimated 42 neurosurgery centers, 1500 neurosurgery beds, and 150 qualified practicing neurosurgeons in Pakistan.[1] Considering that Pakistan has an estimated population of 170 million, this gives a ratio of 1 neurosurgeon/1,130,000, which is a very small number compared to the industrialized countries. The worldwide ratio is one neurosurgeon / 230,000 people; Africa has a ratio of one neurosurgeon / 1,352,000 people, while the ratio is 1 neurosurgeon / 121,000 people in Europe, and one neurosurgeon / 81,000 people in North America.[]ㅡ

Maintaining high standards in neurosurgical training has been a topic of debate, especially in developing countries like Pakistan. $[\underline{6}, \underline{10}]$ The present study was undertaken to observe interdepartmental variation in the availability of resources and academic activities within various neurosurgery programs of Pakistan in an attempt to make recommendations for improvement, and highlight deficiencies. 
This was a proforma-based (attached as a link) survey of neurosurgery trainees and young neurosurgeons of Pakistan. The proforma was mailed to a total of 48 people, and was filled by 36 respondents from 11 neurosurgery centers of the country (response rate 75\%). This sample consisted of trainees from neurosurgery training programs in all the four provinces of the country. All centers were accredited for neurosurgery training in Pakistan. The data were later analyzed by using the Statistical Package for Social Sciences (SPSS) version 13.0 (Chicago, IL, USA).

\section{RESULTS}

Out of 36 respondents, 33 were male and three were female trainees. Eighty-three percent $(n=30)$ of the respondents were doing their training requirement for the Fellowship program (FCPS) offered by the College of Physicians and Surgeons of Pakistan (CPSP). The rest $(n=6)$ of the respondents were working in different centers, to get a Masters of Surgery (MS) qualification in neurosurgery from their respective universities. The mean age of the trainees was 31 years (range $24-36$ years) and the mean duration of training was 3.8 years at the time of survey.

Eighty percent $(n=29)$ of respondents had more than one English neurosurgery textbook in the department. The most frequently consulted textbook was the Youman's Textbook of Neurosurgery, which was generally considered a suitable guide for neurosurgery residency / Fellowship. Other common texts were Schmidek and Sweet's, 'Operative Neurosurgical techniques: Indications, Methods, and Results,' and Greenberg's, 'Handbook of Neurosurgery'. The majority of centers ( $80 \%$ ) had access to at least one neurosurgery journal, but only $40 \%$ had subscription to more than one international indexed journal. The most frequently read journal was, the Journal of Neurosurgery (JNS). Another constraining factor noted in a majority of neurosurgical centers

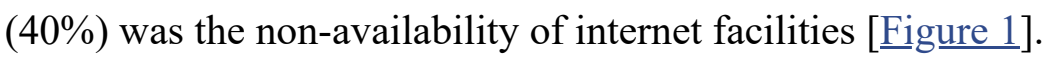

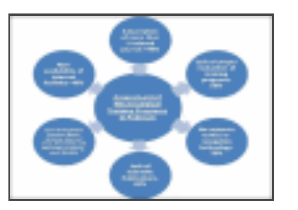

\section{Figure 1}

Assessment of neurosurgical training programs in Pakistan

There was no uniform pattern for academic sessions in the different centers. Thirty percent of the programs were not conducting any teaching sessions for the basic sciences, and $40 \%$ of the centers had no regular journal club meetings or literature reviews. Similar deficiencies were noted in morbidity and mortality ( $\mathrm{M}$ and $\mathrm{M})$ meetings and neuroradiology and neuropathology sessions [Figure 2]. Eighty percent of the respondents reported attending interdepartmental and city-wide meetings, $85 \%$ had no scientific publications in national or international journals, and $35 \%$ of the respondents reported delivering presentations at national or international neurosurgery meetings.

\section{Figure 2}




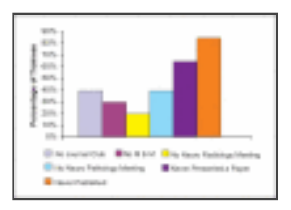

Academic shortcomings

Fifty percent of the respondents reported an evaluation of their training program by national and international faculty at intervals of every two years or longer.

The lack of cadaveric dissection laboratories was another aspect of concern in $95 \%$ of the respective centers. It was a significant observation that only $5 \%$ of the centers made use of dissection laboratories to practice on a cadaveric specimen. We also looked into the availability of different modern surgical tools in the neurosurgical armamentarium of the participating centers. This revealed that the majority of trainees $(70 \%)$ had no exposure to frameless or frame-based navigational technology in routine neurosurgical procedures. Only $60 \%$ of the respondents reported exposure to endoscopic surgeries.

\section{DISCUSSION}

Dr. W. Halsted was the first to introduce a formally structured residency program in the USA, at the John Hopkins Medical School, at Baltimore, in the early twentieth century. However; there are many other formats that have been introduced since. As a result, there are significant differences in the national, regional, and international training programs around the world.[요, $\underline{12}]$ Therefore, these programs need constant re-evaluations in order to improve the standard of neurosurgical education.[16] Neurosurgery, as a surgical discipline of its own right, in Pakistan, began with the arrival of Omar Vali Jooma at the Jinnah Postgraduate Medical Center, in Karachi, in September 1951.[1] Five years later, Brigadier G. D. Qazi joined him as Pakistan's second neurosurgeon, when he established Pakistan's second neurosurgery center at the Combined Military Hospital, Lahore. Pakistan's third neurosurgeon was Bashir Ahmad who arrived here in 1963, and established a neurosurgery center at the Nishtar Medical College and Hospital, Multan, where he practiced for four years before shifting to King Edward Medical College and the associated Lahore General Hospital. This marked a new era for neurosurgery in the province of Punjab, Pakistan's largest and most populous province. Lahore General Hospital, in the years to come, became one of the busiest neurosurgery centers in the world.

Since then, neurosurgery in Pakistan, in general, and neurosurgical training, in particular, have evolved tremendously.

There are two coexisting neurosurgery licensing bodies with separate training formats, in Pakistan. The more popular of the two is the CPSP as it is much more widely recognized. The CPSP runs a Fellowship program at the end of which the trainee is awarded the FCPS diploma, and the other is the university-based Master of Surgery program, at the end of which the trainee is awarded an MS degree. On an average, these programs require five to six years of supervised neurosurgery training, at the end of which the trainees are licensed to practice as independent consultants. Every year, around four to eight postgraduate trainees fulfill the 
requirements and complete their training from these two programs. This small number of neurosurgeons added to the list every year does not match the need for consultants in the country.

At most institutions, neurosurgery training is a six-year program, which involves general surgery training in the first two years and focused neurosurgical training in the next four years. During their training, the trainees are also required to rotate in the Ear, Nose, and Throat (ENT), Anesthesia, Ophthalmology, Radiology, and Neurology Departments. To successfully complete their postgraduate training, the trainees also have to complete an independent research project and pass the examination conducted by CPSP.

Both of these programs have rigid guidelines, to ensure that the training is monitored and fulfills certain requirements. CPSP itself evaluates the standard of training and facilities available at the accredited institutions. However, the requirements focus primarily on the basic fundamentals of neurosurgery, which include learning how to perform basic neurosurgical procedures, completing the specified length of time in different rotations, and so on, rather than attempting to achieve high standards of training, by focusing on a certain complexity of cases observed and performed by trainees, the ability of trainees to perform cases independently, and the exposure to new surgical techniques and instruments. Therefore, the concern is that currently even a center with only modest faculty and equipment and no research infrastructure can still qualify to train clinical neurosurgeons. This may be appropriate at present, as there is an acute shortage of both neurosurgeons as well as resources. However, the question remains: Is this a recipe for success for the future?

The present study is an attempt to highlight the considerable and disturbing large variations in the standard of training at the different centers of Pakistan. A questionnaire-based study was conducted, structured over the responses of neurosurgery trainees and young neurosurgeons in the country. Therefore, despite representations from all four provinces, it may not fully represent the current state of neurosurgery training in the entire country. However, the results should be taken seriously as they show gross differences in the working structure of departments, the availability of resources, and the types of exposure of trainees. All these directly affect the standard of training acquired by graduating from these programs. One of the most disturbing results of the current study is the lack of internet access experienced by almost $40 \%$ of the respondents. Therefore, trainees cannot have access to online journals and books. Of further concern is the fact that there is only a rarified educational program evaluation of more than half the centers by an external governance body (comparable to the Accreditation Council for Graduate Medical Education (ACGME )). It is a surprising fact that more than one-third of the trainees reported not having regular $\mathrm{M}$ and $\mathrm{M}$ meetings at their center, a mandatory meeting in most internationally renowned centers interested in evaluation and improvement of their standard of care. Also of concern is the fact that more than $90 \%$ of the respondents reported 'no' to having access to cadaveric or skills laboratories and almost $85 \%$ of the respondents reported not having a publication to their credit; $65 \%$ reported not having presented at any national or international level in their careers. It is well-recognized that such a lack of research exposure results in young neurosurgeons distancing themselves from substantial research advances and technological advances, which is now considered essential to become an academic surgeon and future leader.[3,ㅆ] In case of pursuing an academic career, research at an 
early point is considered a must and is facilitated in most of the European and North American academic centers, as highlighted by Burkhardt and colleagues.[4]

Some of the practical shortages of the clinical activities of our training programs, such as, the absence of neuronavigation facilities and endoscopy and cadaveric laboratories, may be attributed to lack of resources, which is a common problem for institutions in Pakistan. Although a number of workshops are conducted for teaching different surgical techniques, including spine surgery and instrumentation, their usefulness is questionable as many institutions do not have the resources or instruments for their trainees to practice what they have learnt theoretically. Even sub-disciplines like stereotactic radiosurgery / neurosurgery, functional neurosurgery, epilepsy surgery, and movement disorder surgery are under-represented in Pakistan. Despite these deficiencies, one of the strengths of many neurosurgery training programs is the support from other related specialties like Neurology, Radiology, ENT, Ophthalmology, Anesthesia, and Rehabilitation. The support is maintained by frequent consultation and coordination among the consultants of these specialties. Apart from the lack of resources, political unrest in the country has also contributed to the deficiencies of the training programs in the country, as it creates a hindrance in the training and modifies the attitude of trainees. It disrupts their training curriculum as well as poses problems in obtaining visas and visiting other countries. The political situation also makes it difficult to organize conferences and workshops as guest lecturers and professors are also reluctant to attend these meetings.

Lack of continuing research in the academic institutions of Pakistan has also been criticized and can partly be attributed to lack of funding.[13] However, other core deficiencies, such as regular morbidity meetings, journal clubs, conduction and presentation of research, multispecialty meetings, as well as, rotations, and even the availability of the internet do not require large resource allocations or drastic changes in the system, and can be implemented easily. Although a neurosurgery trainee has a busy schedule throughout the day, it should not be too difficult to allocate one to two hours some time during the week for an academic session in the training curriculum, without actually compromising on the surgical exposure the trainees get. Similarly, regular program evaluation requires establishment of a system (internal or external) of audit and self-improvement, which again does not require excessive funding or time.[]]

Pakistan's first neurosurgery research article was published in 1979, in which I. H. Bhatti reported a preliminary study looking at platelet adhesiveness in spontaneous subarachnoid hemorrhage, the final results of which were never published.[ㄹ] In a recent review of publications, it was concluded that no level 1 or 2 evidence was contributed by neurosurgeons in Pakistan in the last six years.[14] Publications were few in number, consisting mainly of case reports and case series and came from just a few authors situated at select centers.[14] To this day, Pakistan does not have a scientific peer-reviewed journal, indexed with Pubmed or dedicated to neurosciences research.[9] Although there are now four Pakistani medical journals indexed with the National Library of Medicine (NLM), the Journal of Pakistan Medical Association (JPMA) and the Journal of College of Physicians and Surgeons Pakistan (JCPSP) have been the oldest and most popular within the local neurosurgery community. Two neurosurgery journals, Pakistan Journal of Neurological Sciences (PJNS) and Pakistan Journal of Neurosurgery (official journal of Lahore General Hospital), deal primarily with neurosciences research, but both are in their initial stages and as yet are not indexed with NLM. 
Both the MS and FCPS programs require completion of a research project and submission of a thesis for eligibility of a license. Currently, the Pakistan Society of Neurosurgeons holds regular annual meetings as well as a Neurotrauma Conference every alternate year, which invites article presentations well in advance and encourages trainees and young neurosurgeons, through free traveling and lodging arrangements and medals and awards for best presentations. The Young Neurosurgeons Forum also awards the best research articles every year. International organizations like the Asian Congress of Neurological Surgeons (ACNS) and World Federation of Neurosurgical Societies (WFNS) similarly encourage the participation of young neurosurgeons in their conferences, especially from developing countries, through traveling Fellowships. Yet, almost 65\% of the senior neurosurgery trainees (mean year in training 3.8) report not having made any national or international presentations. Those who participate in these conferences either belong to a few institutions where research is given importance and funds are available or they are the ones who can personally fund their expenses of traveling and attending such conferences.

It is clear that despite all the ongoing efforts, a research culture is still lacking. Improvement of the quality and number of neurosurgical publications in Pakistan critically depends on the future involvement of trainees and fresh graduates. The workload should not be held solely responsible for these shortcomings as other busy neurosurgery centers in the world manage to entertain a work environment that allows producing a large number of research articles.

In a nutshell, neurosurgical training and practice in developing countries is significantly different from the standards observed in developed countries. Due to lack of resources and constraints by the health budget, several of the universally advertised resources in the field of neurosurgery might not be first line or even second line of demand in developing countries.

We recognize the current limitations of the existing healthcare system in Pakistan, which precludes comparably high standards of training across various centers. However, several deficiencies observed in the current study can be corrected by simple measures, such as, providing internet access and online journal subscriptions, promoting research, organizing $\mathrm{M}$ and $\mathrm{M}$ meetings, journal clubs, interdepartmental meetings, and so on. To allow for significant clinical improvement, we recommend the development of regular exchange programs between centers, to allow trainees to gain first-hand exposure to facilities not available in their center of training.

\section{REFERENCES}

1. Alphen HA. World Federation of Neurosurgical Societies 1955-2005: A history. 2000:206-7.

2. Bhatti IH, Patel YM. Platelet adhesiveness in spontaneous subarachnoid haemorrhage: A preliminary study. J Pak Med Assoc. 1979;29:2-5. [PubMed]

3. Black PM. Challenges in contemporary academic neurosurgery. Neurosurgery. 2006;58:419-25. discussion 419-25. [ubMed] 
4. Burkhardt JK, Zinn PO, Bozinov O, Colen RR, Bertalanffy H, Kasper EM. Neurosurgical education in Europe and the United States of America. Neurosurg Rev. 2010;33:409-17. [PMC free article] [PubMed]

5. El Khamlichi A. African neurosurgery: Current situation, priorities, and needs. Neurosurgery. 2001;48:1344-7. [ubMed]

6. Enam S. Neurosurgical training in Pakistan. Pak J Neurol Sci. 2007;2:4-5.

7. Godil SS, Kazim SF, Shamim MS. Research-retreat-recovery: A potential model for organization and completion of research projects.Experience from a neurosurgery department in a developing country. Surg Neurol Int. 2010;1:69. [PMC free article] [PubMed]

8. Harbaugh RE, Agarwal A. Training residents in endovascular neurosurgery. Neurosurgery. 2006;59(Suppl 3):S277-81. [PubMed]

9. Kazim SF, Shamim MS. Neuroscience in Pakistan: A neglected avenue. J Coll Physicians Surg Pak. 2010;20:561-2. [PubMed]

10. Ramesh VG. Whither neurosurgical teaching? Neurol India. 2006;54:317-8. [PubMed]

11. Ratcheson RA. Fast forwarding: The evolution of neurosurgery.The 2005 presidential address. J Neurosurg. 2005;103:585-90. [PubMed]

12. Sauvageau E, Hopkins LN. Training in cerebrovascular disease: Do we need to change the way we train residents? Neurosurgery. 2006;59(Suppl 3):S282-6. [PubMed]

13. Shamim MS. Research and publications: Where do we stand? J Pak Med Assoc. 2009;59:62-4. [PubMed]

14. Shamim MS, Enam SA, Kazim SF. Neurosurgical research in Pakistan: Trends of publication and quality of evidence. Clin Neurol Neurosurg. 2011;113:107-10. [PubMed]

15. Stone JL, Vilensky J, McCauley TS. Neurosurgery 100 years ago: The Queen Square letters of Foster Kennedy. Neurosurgery. 2005;57:797-808. discussion 797-808. [PubMed]

16. Sure U, Miller D, Bozinov O. Neurosurgical training in Europe, problems and possible solutions. Surg Neurol. 2007;67:626-8. discussion 628-33. [PubMed]

$2011 ; 2: 183$

Published online 2011 Dec 26. doi: 10.4103/2152-7806.91138

\section{A Neurological Surgeon in Pakistan - August to December, 1962}


While visiting the 'Sub Continent' of Asia, the Indiana University Chancellor, Herman B. Wells, offered West Pakistan help in developing additional Medical Schools, as only one was left in there after it had been separated from India in about 1950. Soon after that the Pakistani Physicians arrived on the Indiana University Medical School (IUMC) Campus, to become better acquainted in teaching Pre-clinical subjects. After several of those physicians returned to Pakistan and started teaching Medical courses, requests were made for Teachers of Clinical subjects, and eventually Residency experiences. I was asked to spend several months learning what would be needed to establish speciality training in the new Medical School Hospitals being established throughout what was then East and West Pakistan, that is, before East Pakistan became Bangladesh.

My plane arrived one late afternoon in Karachi, West Pakistan, and I was taken to my quarters near the Pre-clinical part of the recently-built Medical School and older adjacent hospital. Soon after my arrival, I was invited to have dinner with the family of an IUMC teacher of physiology, who was helping to establish the pre-clinical part of the Medical School, and also to establish a serious interest in herpetology and the development of snake anti-venoms. While waiting for dinner, one of the young daughters moved my arm off the arm of the chair, and replaced it with a short fat snake, unlike anything I had seen before. She explained that that particular snake was a family pet, as she showed me how to pet it. Dinner was excellent India-style fare, and I had a restful night after a long day of travel.

The next day a British-trained neurosurgeon, Omar Jooma, MD, took me on rounds of several 20-bed wards with a variety of well-cared-for neurosurgical and general surgical patients. In a few days, I was deep into the neurosurgery schedule myself, with Dr. Jooma, but more often by myself, assisted by one or another of the younger general surgeons.

The operating room, was large with a wooden floor and light-colored painted walls and a large open window, which overlooked a courtyard where the patients' and their families' horses and donkeys were kept. Braying and scuffling animal feet were heard through the open window, particularly in the early mornings, before they were fed. After operating there for a few days I persuaded the nurses, who were very good and attentive, to move the head or back to be operated on away from the open window to the other side of the room. Quite adequate operating room (OR) lights could be moved around on castors to provide good illumination of the operative sites. There was no air-conditioning, so the nurses would wait until the surgery progress was such that they could put a towel wrung out in ice water over the surgeon and his helper's backs for a few minutes of wonderfully cool comfort. In spite of these unusual means of completing surgeries, neither I nor Dr. Jooma had any wound infections.

Dr. Jooma and I each performed many operations, but I will mention a few that I remember clearly, with the understanding that there were many more. A young man had been carried piggy-back almost a thousand miles from the Himalayan mountains in the north of Karachi. I have no recollection of the diagnosis, but I remember removing a well-encapsulated abscess arising from his mastoid that compressed one of his temporal lobes. A short while post the operation he was able to walk joyously back toward his mountain home. I never saw or heard from him after he walked off from the hospital ward, and had to assume he remained well 
enough to help care for his family's farm. He also walked off the ward among his many relatives. Length of hospital stay seemed never to be an issue.

Another similar case had a medium-sized acoustic neurinoma removed, without the use of magnification or other current amenities. He also walked off the 20-bed ward without difficulty. I remember one elderly gentleman, from whom a benign spinal tumor was removed, sitting up on his bed, both pre- and post-operation, while one or the other of his sons squatted behind him rubbing his back.

The patients' families brought their own bedding, and convenient places were supplied for relatives to wash, and clothes lines were provided to dry their clothes quickly in the warm sun. It was impossible to count the number of relatives attending each patient, as they provided much of the care except for medication or vital sign recording. Relatives cooked the best-liked meals of the patients on small stoves, on the balconies surrounding each ward, and fed them, making a real family affair of each hospitalization. When x-rays were needed, the families were told how many and what sizes were needed, so relatives could go into the city to buy and bring them back to be properly exposed by the x-ray technicians.

The time came, after I had become somewhat accustomed to this routine, for me to visit other sites where new medical schools were being developed in both East and West Pakistan. The first was King Edward Hospital in Lahore, West Pakistan, many miles north of Karachi, where Dr. Bashir Amid, who had seven years of neurosurgical training in one of the world-renowned neurosurgical centers had started to practice. During training, Dr. Bashir spent all the money he earned buying neurosurgical instruments and sending them back to his home in Lahore, so only a few days after he completed his training he was able to start operating. Other neurosurgeons with similar training had to wait months after arriving for sufficient instruments to start operating. Dr. Bashir and I made a lasting friendship, as we discussed Pakistan's medical needs, particularly with regard to resident training. He had a strong reputation for his diagnostic and surgical skills, and eventually became the principal of the enlarged King Edward Hospital Medical School Complex.

My next visit was to Peshawar. Work on the building for the new medical school had not yet started, and the elderly surgeons I met there were not sure if neurosurgery should be a separate discipline from general surgery or resident training. I assumed, but had not confirmed that this new medical school was one of the ten that Pakistan was planning. I visited several other locations, one of which was Abbottabad, where Osama Bin Laden was found during the last few days of April 2011. My only recollection of that stop was a walled courtyard full of beautiful little black and white goat kids frolicking.

On my way back to the USA I landed in the city of Dakar, what was then East Pakistan, now Bangladesh, where a new preclinical medical school had opened, essentially only classrooms and laboratories. The friendly professor who showed me around learned that my father was one of the early leperologists, and suggested that I visit a leper community on the Changregoona River, not far east of Dakar. I got up early on a Sunday morning and caught a launch to take me to visit British missionaries who cared for the lepers in this community. The launch went slowly against the flow of the river, stopping at many docks to load and unload passenger, animals, and various bundles of goods. When we reached the landing for the leper community, I was directed to the 
mission house. I found no one was home, so I wandered around the leper village, watching women making cloth and men doing other chores. I recognized the signs of leprosy among the friendly people, whose children seemed to be uninfected. The British missionaries shared their lunch with me and arranged for a three-wheeled taxi to take me back to Dakar. On the way back to the USA I stopped in Burma, now Myanamar, where I was privileged to sail a 'lightening'-styled sail boat, Bangkok, and then went on to the Large Island of Hawaii, where my family joined me on Kalikakua Bay, where Captain Cook was boiled and the volcano started pouring out red hot liquid rock two days before we were scheduled to leave.

Footnotes

Go to:

Available FREE in open access from: http://www.surgicalneurologyint.com/text.asp? 2011/2/1/183/91138

Articles from Surgical Neurology International are provided here courtesy of Wolters Kluwer -- Medknow Publications 